\title{
Magnetic activities on young stars
}

\author{
Kenji Hamaguchi, Yohko Tsuboi, Kensuke Imanishi, and Katsuji Koyama \\ Department of Physics, Faculty of Science, Kyoto University, Kitashirakawa-oiwakeho, Sakyo, Kyoto 606-8502, Japan
}

(Received June 20, 2000; Revised November 1, 2000; Accepted November 29, 2000)

\begin{abstract}
Almost all young stellar objects (YSOs) are now known to emit X-rays driven by the magnetic activity. The solar-type dynamo is the plausible mechanism to generate the magnetic fields, but it does not work properly on protostars or intermediate-mass YSOs due to the difference of their stellar inner structure. To find an alternative mechanism, the structure and energy of magnetic fields surrounding stars should be investigated. In this sence, a stellar X-ray flare gives us important information because it directly traces an activity of one magnetic loop. With this motivation, we searched for stellar X-ray flares using the ASCA satellite and detected seven flares from low-mass and intermediate-mass YSOs. By comparing flare parameters with the absorption column density $\left(N_{\mathrm{H}}\right)$, we found that younger stars tend to have higher plasma temperature and larger emitting volumes. Furthermore, the flare loop size should be far larger than the solar radii in low-mass protostars and Herbig Ae/Be stars (HAEBEs). These facts suggest that the magnetic fields of low-mass protostars and HAEBEs far extend from the stellar main body and probably connect to the circumstellar disks, and that the magnetic reconnection by the star-disk differential rotation should energize the X-ray flares. We also present supporting evidence of quasi-periodic flares detected on the ClassI protostar YLW15.
\end{abstract}

\section{X-Ray Properties of Young Stars}

The initial phase of low-mass stars called protostars form in the deep inside of molecular clouds by the self-gravitation of interstellar gases. After the dynamical accretion stops by disappearing of the gas envelopes, they move to the classical T-Tauri stars (CTTSs), in which they gradually increase the weight by occassional mass accretion from circumstellar disks, and the stellar cores contract quasi-statically. Then, the circumstellar disks are exhausted and lost, and it is classified to weak-lined T-Tauri stars (WTTSs). When the stellar core have enough temperature to ignite the nuclear burning, they move to the main-sequence phase.

One of splendid discoveries of the Einstein satellite (19781981) was that CTTS and WTTS show powerful X-ray activities. The X-ray monitoring of several star forming regions, $\rho$ Ophiuchi, Taurus and so on, revealed dramatic X-ray variability on these stars, which Montmerle et al. (1983) called "X-ray Christ-mass tree"1. They show occassional flares with fast rise and exponential decay. These features are quite similar to solar ones, but the luminosity could be $10^{4}$ times as high as the solar ones (see Table 1). X-rays from low-mass protostars had not been detected since protostellar X-rays, even if it exists, suffer deep absorption from the surrounding molecular clouds. The Japanese X-ray satellite ASCA, which was launched in 1993, has an imaging capability in hard X-ray band to see through the molecular cloud, and dis-

\footnotetext{
${ }^{1}$ Results of the long monitoring of the $\mathrm{R} \mathrm{CrA}$ region are shown at http://www-cr.scphys.kyoto-u.ac.jp/member/kenji/ Analysis/RCrA/index.html.

Copy right (C) The Society of Geomagnetism and Earth, Planetary and Space Sciences (SGEPSS); The Seismological Society of Japan; The Volcanological Society of Japan; The Geodetic Society of Japan; The Japanese Society for Planetary Sciences.
}

covered X-rays from protostar clusters in R Corona Australis (R CrA) molecular cloud (Koyama et al., 1996). The luminosity was still higher than those of TTSs (see Table 1), and they also exhibited powerful X-ray flares. From these results, low-mass stars from protostar to the main-sequence are now widely understood as showing magnetic activities.

On the other hand, magnetic activities on intermediate and high mass stars had not been predicted. This is because they do not have surface convection zone needed to drive the dynamo mechanism. Massive main-sequence stars earlier than B5, whose masses are typically larger than 10 $\mathrm{M}_{\odot}$, have a mechanism to emit soft X-rays by UV driven stellar wind shocks (see Table 1), but younger stars do not have enough UV field to accelerate the stellar wind, and hence are not thought to show any X-ray emission. However, ROSAT detected X-rays from not a few intermediate-mass YSOs called Herbig Ae/Be stars (HAEBEs, Zinnecker and Preibisch, 1994) equivalent to low-mass TTSs. Successive ASCA observations have revealed that some of HAEBEs have high temperature plasma of about $3 \mathrm{keV}$ (e.g. Skinner and Yamauchi, 1996; Yamauchi et al., 1998), which cannot be produced by slow stellar wind of HAEBEs (below 500 $\mathrm{km} / \mathrm{s}$ ). Several IR observations also report jets and outflows around HAEBEs, that are relics of dynamic activities on the stellar cores. These HAEBE activities have long been puzzling. In a recent ASCA data analysis, we discovered a large X-ray flare from the Herbig Be star MWC297 (Hamaguchi et al., 2000). This result would be important because it indicates the magnetic activity on HAEBEs and explains the high energy phenomena.

Most PMSs in a wide mass range seem to show magnetic activities. However, the magnetic amplification mechanism of protostars and HAEBEs is still unclear. The inside of pro- 
Table 1. The X-ray properties and typical stellar parameters.

\begin{tabular}{lccccccc}
\hline & $\begin{array}{c}\mathrm{kT} \\
(\mathrm{keV})\end{array}$ & $\begin{array}{c}N_{\mathrm{H}} \\
\left(10^{22} \mathrm{~cm}^{-2}\right)\end{array}$ & $\begin{array}{c}\log \left(L_{\mathrm{X}}\right) \\
\left(\mathrm{ergs} \mathrm{s}^{-1}\right)\end{array}$ & Var. & Sp. & $\begin{array}{c}\text { Mass } \\
\left(\mathrm{M}_{\odot}\right)\end{array}$ & $\begin{array}{c}\log (\text { Age }) \\
(\mathrm{yrs})\end{array}$ \\
\hline The sun & $<1$ & - & 27 & $\mathrm{y}$ & $\mathrm{G} 2$ & 1 & 9.7 \\
TTS & $\sim 1$ & $0-2$ & $28-30$ & $\mathrm{y}$ & $\mathrm{G} / \mathrm{K} / \mathrm{M}$ & $\sim 1$ & $6-7$ \\
PS & $\sim 4$ & $\sim 4$ & $30-31$ & $\mathrm{y}$ & $\mathrm{G} / \mathrm{K} / \mathrm{M}$ & $\sim 1$ & $\sim 5$ \\
OB stars $^{1}$ & $<1$ & $\sim 0$ & 31 & $\mathrm{n}$ & $\mathrm{B} 0$ & 10 & $\sim 7$ \\
MWC297 $^{2}$ & 2.7 & 2.4 & 32 & $\mathrm{y}$ & $\mathrm{O} 9$ & $10-26$ & $4.5-5.5$ \\
\hline
\end{tabular}

TTS: T-Tauri stars, PS: Protostars, Var.: X-ray Variability, Sp.: Spectral type.

${ }^{1}$ Typical values of $\mathrm{B} 0$ stars.

${ }^{2}$ Hamaguchi et al. (2000).

Table 2. X-ray flare sources in the R CrA cloud.

\begin{tabular}{|c|c|c|c|c|c|c|}
\hline \multirow{2}{*}{$\begin{array}{c}\text { Source } \\
\text { No. }\end{array}$} & \multirow[t]{2}{*}{ Flare start time ${ }^{a}$} & \multicolumn{2}{|c|}{ Coordinate $(2000)^{c}$} & \multirow[t]{2}{*}{ Source ID } & \multirow[t]{2}{*}{ Source Type $^{d}$} & \multirow[t]{2}{*}{ Detectors $^{e}$} \\
\hline & & R.A. & Dec. & & & \\
\hline 1 & $1994 / 04 / 04,05: 20^{b}$ & $19 \mathrm{~h} 01 \mathrm{~m} 10.84 \mathrm{~s}$ & $-36^{\circ} 47^{\prime} 47.1^{\prime \prime}$ & VSS VIII-27 & IR source & GIS \\
\hline 2 & $1994 / 04 / 08,23: 55^{b}$ & $19 \mathrm{~h} 01 \mathrm{~m} 55.54 \mathrm{~s}$ & $-36^{\circ} 57^{\prime} 31.3^{\prime \prime}$ & $\mathrm{IRS} 9=\mathrm{R} 1$ & protostar & SIS \& GIS \\
\hline 3 & $1998 / 04 / 19,02: 22^{b}$ & $19 \mathrm{~h} 01 \mathrm{~m} 33.65 \mathrm{~s}$ & $-37^{\circ} 00^{\prime} 13.3^{\prime \prime}$ & CrA 1 & WTTS & GIS \\
\hline 4 & $1998 / 04 / 19,13: 28$ & $19 \mathrm{~h} 01 \mathrm{~m} 48.96 \mathrm{~s}$ & $-36^{\circ} 56^{\prime} 17.1^{\prime \prime}$ & IRS6 & CTTS & GIS \\
\hline 5 & $1998 / 10 / 19,20: 38$ & $19 \mathrm{~h} 02 \mathrm{~m} 21.84 \mathrm{~s}$ & $-36^{\circ} 55^{\prime} 35.5^{\prime \prime}$ & $\mathrm{CrA3}$ & WTTS & \\
\hline 6 & $1998 / 10 / 19,15: 31$ & $19 \mathrm{~h} 02 \mathrm{~m} 00.90 \mathrm{~s}$ & $-37^{\circ} 07^{\prime} 41.7^{\prime \prime}$ & V702 CrA & G5 star & GIS \\
\hline 7 & $1998 / 04 / 18,23: 02$ & $18 \mathrm{~h} 51 \mathrm{~m} 08.54 \mathrm{~s}$ & $-36^{\circ} 03^{\prime} 35.3^{\prime \prime}$ & - & - & SIS \& GIS \\
\hline 8 & $1994 / 04 / 12,03: 31^{b}$ & $18 \mathrm{~h} 27 \mathrm{~m} 40.6 \mathrm{~s}$ & $-3^{\circ} 49^{\prime} 50.6^{\prime \prime}$ & MWC297 & O9 or B1.5 & SIS \& GIS \\
\hline
\end{tabular}

${ }^{a}$ The time when the flux rose from the quiescent level.

${ }^{b}$ The start time of the observation.

${ }^{c}$ Typical position uncertainty are $20^{\prime \prime}$ for SIS and $40^{\prime \prime}$ for GIS.

${ }^{d}$ The source type is referred from previous literatures. The evolutional status of some sources are not known.

${ }^{e}$ Detectors which observed the flare. SIS: X-ray CCD camera, GIS: Gas Imaging Spectrometer.

tostars is thought to be fully convective, and there is no anchor point to fix and amplify the magnetic field. HAEBEs have no surface convection zone to drive dynamo mechanism. Standard dynamo theory cannot explain their magnetic activities.

The most direct and undoubted way to solve this problem is to derive the physical parameters of the magnetic field. It is not easy because quiescent X-rays are the assembly of many small flare loops on a stellar surface (e.g. Skinner and Walter, 1998) and plasma parameters calculated from quiescent X-rays are average of enormous number of flare loops. However, there is a good method to observe one flare loop, to detect X-ray flares. From time variations and Xray spectroscopy, we can directly derive plasma temperature and emission measure (E.M.), and estimate electron density, emitting volume and magnetic field strength with few assumptions.

\section{Observations and Results}

We intensively searched for X-ray flares in a low-mass star forming region, the R CrA molecular cloud. ASCA observed this region six times from 1994 to 1998 ( 300 ksec) and found seven flares (see Table 2). As for a flare sample of HAEBEs, we used the MWC297 data. Figure 1 displays flare light curves of the HAEBE MWC297 (left) and the WTTS CrA3 (right). Both variable features are similar to the solar flares with a rapid rise and an exponential decay. We fitted the decay phase with a model of an exponential and a constant component. The decay time scale is shown in Table 3. We then made flare spectra by subtracting the quiescent state spectra as the background. The examples are shown in Fig. 2. All flare spectra are well reproduced by an absorbed thin-thermal plasma model (Raymond-Smith model). The fitting results are also shown in Table 3.

We regard the column density of hydrogen $\left(N_{\mathrm{H}}\right)$ derived from X-ray spectra as stellar ages, but $N_{\mathrm{H}}$ is also affected by the inclination of each star. We compared it with the stellar age of well-known sources and found that younger stars always have larger $N_{\mathrm{H}}$. We henceforth use the $N_{\mathrm{H}}$ value as an indicator of stellar ages. From these values, we evaluate the electron density $\left(n_{e}\right)$ and the volume of the plasma $(V)$ on the assumption of the radiative cooling for flaring plasma; $L_{X}=n_{e}^{2} \Lambda(T) V$ and $\tau_{\text {cool }}=3 n_{e} k T / n_{e}^{2} \Lambda(T)(\Lambda(T)$, the emissivity; $\tau_{\text {cool }}$, the cooling time scale of the plasma). We also derived the magnetic field strength from the equation of 
Table 3. Plasma parameters of the flares.

\begin{tabular}{|c|c|c|c|c|c|c|c|c|}
\hline $\begin{array}{c}\text { Source } \\
\text { No. }\end{array}$ & $\begin{array}{c}\tau_{\text {cool }} \\
\left(10^{3} \mathrm{sec}\right)\end{array}$ & $\begin{array}{c}N_{\mathrm{H}} \\
\left(\mathrm{cm}^{-2}\right)\end{array}$ & $\begin{array}{c}k \mathrm{~T} \\
(\mathrm{keV})\end{array}$ & $\begin{array}{c}\mathrm{L}_{X} \text { peak }^{a} \\
\left(10^{30} \mathrm{ergs} \mathrm{s}^{-1}\right)\end{array}$ & $\begin{array}{c}E . M . \\
\left(10^{54} \mathrm{~cm}^{-3}\right)\end{array}$ & $\begin{array}{c}\mathrm{n}_{e} \\
\left(10^{11} \mathrm{~cm}^{-3}\right)\end{array}$ & $\begin{array}{c}\mathrm{V} \\
\left(10^{31} \mathrm{~cm}^{3}\right)\end{array}$ & $\begin{array}{c}\text { B } \\
\text { (gauss) }\end{array}$ \\
\hline 1 & 4 & 0.3 & 4.0 & $40^{b}$ & 12 & 4.8 & 0.8 & 340 \\
\hline 2 & 25 & 11.0 & 11.4 & $90^{b}$ & 26 & 1.4 & 17.3 & 300 \\
\hline 3 & 3 & 0.0 & 3.3 & $27^{b}$ & 11 & 5.3 & 0.6 & 320 \\
\hline 4 & 13 & 5.5 & 4.1 & $50^{b}$ & 21 & 1.5 & 14.0 & 190 \\
\hline 5 & 18 & 0.3 & 4.3 & $32^{b}$ & 5.8 & 1.1 & 7.4 & 170 \\
\hline 6 & 12 & 0.0 & 4.2 & $5.8^{b}$ & 1.4 & 1.7 & 0.8 & 200 \\
\hline 7 & 7 & 0.1 & 2.4 & $9.6^{b}$ & 4.0 & 2.3 & 1.3 & 180 \\
\hline 8 & 56 & 2.5 & 6.7 & $450^{c}$ & 35 & 0.44 & 1800 & 130 \\
\hline
\end{tabular}

${ }^{a}$ The peak luminosity of the flare between $0.8 \mathrm{keV}$ and $10 \mathrm{keV}$.

${ }^{b}$ The distance is assumed to be $130 \mathrm{pc}$.

${ }^{c}$ The distance is assumed to be $450 \mathrm{pc}$.

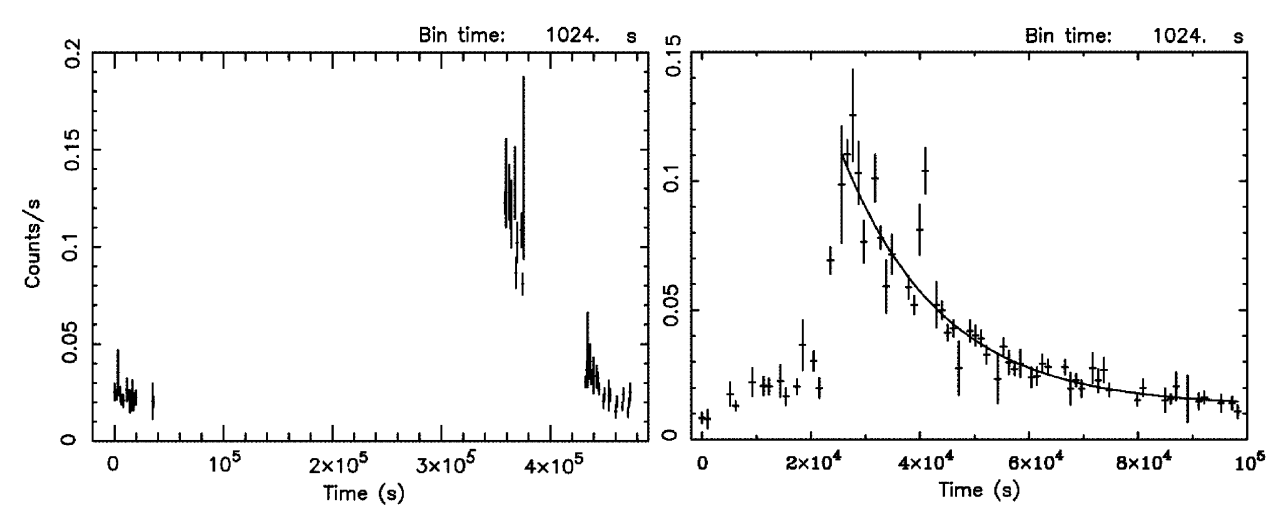

Fig. 1. Flare light curves of MWC297 (HAEBE: left) and CrA3 (WTTS: right).

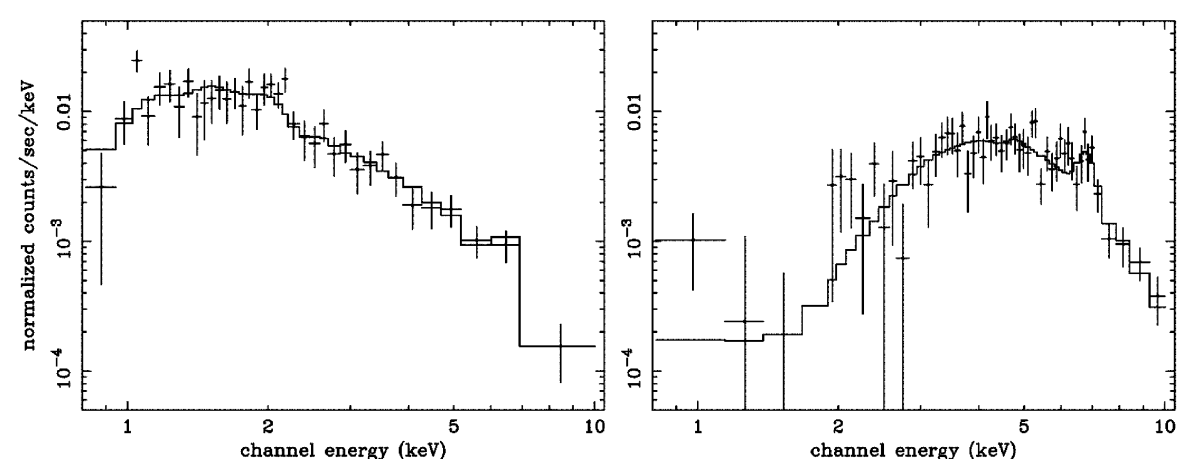

Fig. 2. Flare Spectra of CrA3 (WTTS: left) and R1 (protostar: right). Large absorption in the soft X-ray band is clearly seen in the right spectrum.

$B=\left(12 \pi n_{e} k T\right)^{1 / 2}$, assuming that the magnetic field energy released is completely converted to the thermal one.

Figure 3 displays five graphs comparing the above mentioned quantities against $N_{\mathrm{H}}$. The number of the flare samples we analysed is insufficient to deduce the evolution of these parameters. We then added more flare samples on several publications (V773 Tau in Tsuboi et al., 1998; $\rho$ Ophiuchi dark cloud in Kamata et al., 1997 and Tsuboi et al., 2000; SSV63E+W in Ozawa et al., 1999). The top panel shows $N_{\mathrm{H}}$ versus plasma temperature (left panel) and E.M. (right panel). Flares above $5 \mathrm{keV}$ were not seen in lower $N_{\mathrm{H}}(<1-$ $2 \times 10^{22} \mathrm{~cm}^{-2}$ ) sources, whereas some sources with $N_{\mathrm{H}}>1-$ $2 \times 10^{22} \mathrm{~cm}^{-2}$ show very high temperature flares of about $10 \mathrm{keV}$. E.M. is larger for high $N_{\mathrm{H}}$ and seems to have a 

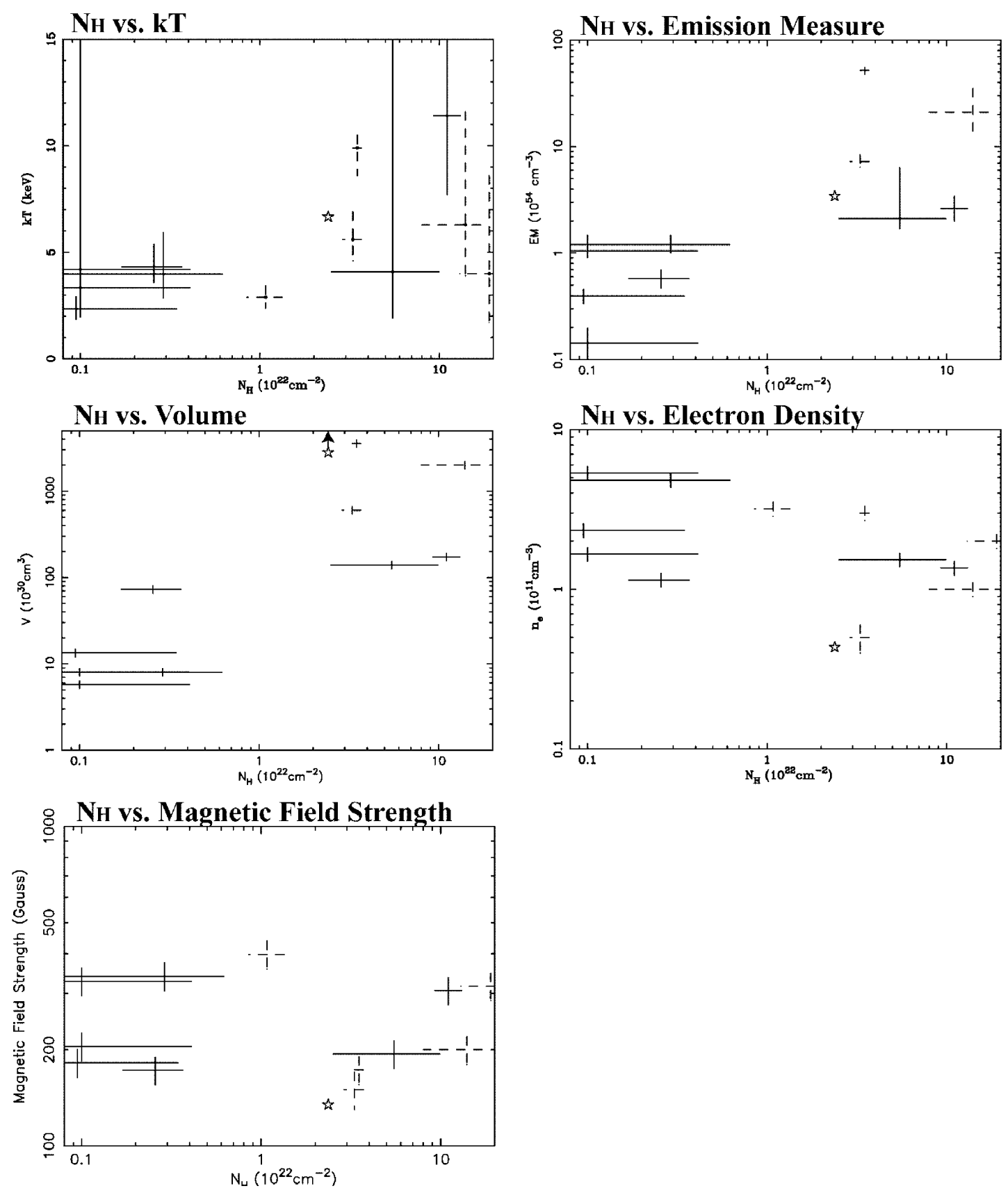

Fig. 3. $N_{\mathrm{H}}$ vs. several flare parameters. Solid crosses indicate sources in the R CrA region whereas dashed crosses do sources referred from several papers, and a star mark does MWC297.

transition at $N_{\mathrm{H}} \sim 1-2 \times 10^{22} \mathrm{~cm}^{-2}$. It means that plasma heated up by flares cool down by X-ray radiation. Electron density (center right) seem to be constant with $\sim 1-5 \times 10^{11}$ $\mathrm{cm}^{-3}$, whereas emitting volumes (center left) are quite high for younger stars. The magnetic field strength is between $100-400 \mathrm{G}$ and does not vary with the stellar age.

\section{Discussion}

Temperature, E.M. and emitting volume seem to have a transition at $N_{\mathrm{H}} \sim 1-2 \times 10^{22} \mathrm{~cm}^{-2}$, which suggests that magnetic structure of low-mass stars changes at this stellar age. For large $N_{\mathrm{H}}$ corresponding to the protostar phase, the typical length of the emitting loops $\left(V^{1 / 3}\right)$ is significantly larger than the stellar radius (solar radius $\sim 7 \times 10^{10} \mathrm{~cm}$ ).
Therefore, we have to consider the flare loop structure far larger than the stellar size. One of the most plausible models is the magnetic structure connecting between a star and a disk.

Supporting evidence would be derived from quasi-periodic X-ray flares of protostar YLW15. The left panel of Fig. 4 shows a light curve of YLW15 obtained with ASCA (Tsuboi et al., 2000). In this observation, YLW15 showed three continual X-ray flares every 20 hours. This event can be explained by the sequence as follows. The right panel of Fig. 4 is an schematic picture of the magnetic structure of YLW15 presented by Montmerle et al. (2000). The central circle is a protostellar core, surrounding annulus is an accretion disk and the magnetic field is shown by solid lines. We assume 

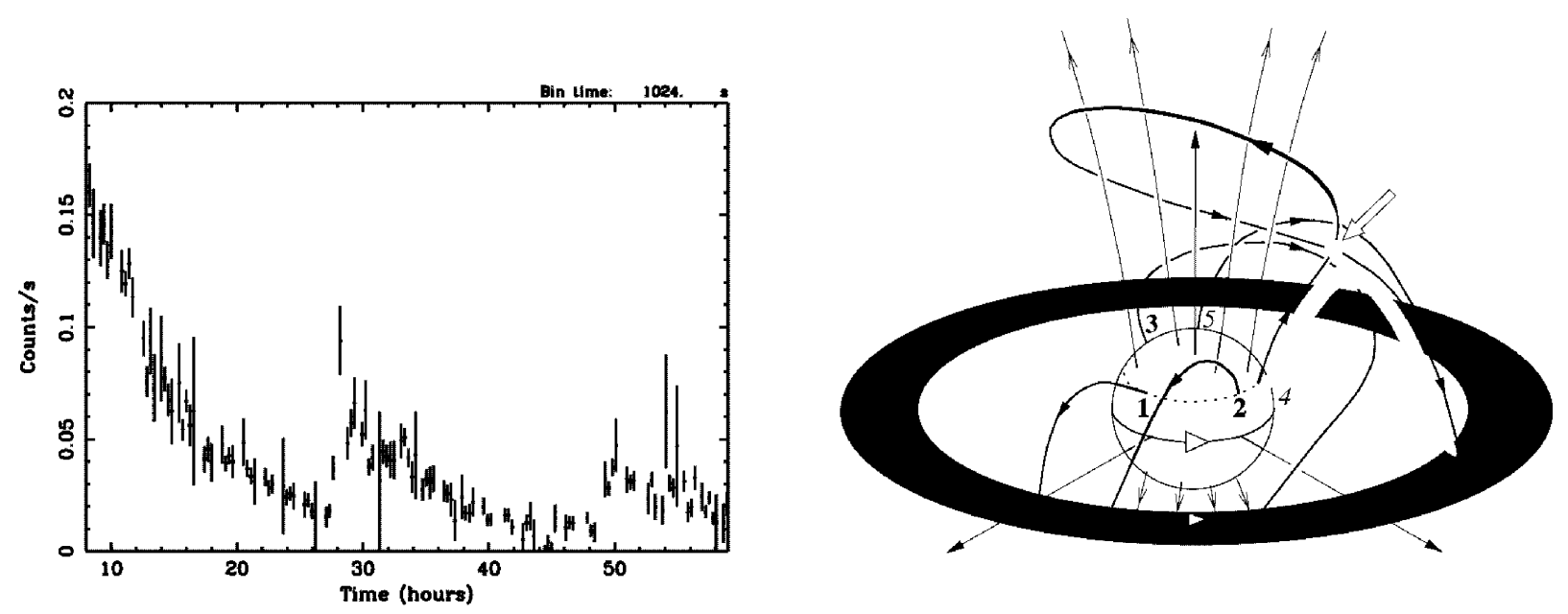

Fig. 4. Left: A flare light curve of YLW15 taken by an ASCA observation in March 1997 (Tsuboi et al., 2000). Horizontal axis is a time, and vertical axis is a linear scale of detector count rate. Right: Schematic view of possible magnetic configuration quoted from Montmerle et al. (2000). The solid lines describe five steps of magnetic fields changing in a sequence of Nos. 1-5 as rotating difference is enhanced between a star and a disk.

that the magnetic field connecting between star and disk is generated on No. 1. Then it is gradually twisted by the differential rotation between star and disk (Nos. 2-3). After the one rotational difference, magnetic field will reconnect and burst out an X-ray flare (No. 4). In the ASCA observation, we might see three rotational periods of differential rotation. This model can solve the problem of amplification mechanism of magnetic fields on protostars and HAEBEs. The X-ray emission mechanism could be the same in the youngest phase of stars independent of their masses.

Acknowledgments. The authors are grateful to anonymous referees for their usuful comments.

\section{References}

Hamaguchi, K., H. Terada, A. Bamba, and K. Koyama, Large X-ray flare from the Herbig Be star MWC 297, ApJ, 532, 1111, 2000.

Kamata, Y., K. Koyama, Y. Tsuboi, and S. Yamauchi, X-ray analysis of the $\rho$ Ophiuchi dark cloud with ASCA: Source identification, X-ray spectra, and temporal variability, $P A S J, 49,461,1997$.

Koyama, K., K. Hamaguchi, S. Ueno, N. Kobayashi, and E. D. Feigelson, Discovery of hard X-rays from a cluster of protostars, PASJ, 48, L87, 1996.

Montmerle, T., L. Koch-Miramond, E. Falgarone, and J. E. Grindlay,
Einstein observations of the Rho Ophiuchi dark cloud-an X-ray christmas tree, ApJ, 269, 182, 1983.

Montmerle, T., N. Grosso, Y. Tsuboi, and K. Koyama, Rotation and X-ray emission from protostars, $A p J, \mathbf{5 3 2}, 1097,2000$.

Ozawa, H., F. Nagase, Y. Ueda, T. Dotani, and M. Ishida, Detection of hard X-rays from a class I protostar in the HH 24-26 region in the Orion molecular cloud, ApJ letter, 523, L81, 1999.

Schmitt, J. H. M. M. and F. Favata, Continuous heating of a giant X-ray flare on Algol, Nature, 401, 44, 1999.

Skinner, S. L. and F. M. Walter, ASCA observations of the T Tauri star SU Aurigae and the surrounding L1517 dark cloud, ApJ, 509, 761, 1998.

Skinner, S. L. and S. Yamauchi, ASCA observations of HD 104237 (A4e) and the origin of X-ray emission from Herbig Ae stars, ApJ, 471, 987, 1996.

Tsuboi, Y., K. Koyama, H. Murakami, M. Hayashi, S. Skinner, and S. Ueno, ASCA detection of a superhot 100 million K X-ray flare on the weak-lined T Tauri star V773 Tauri, ApJ, 503, 894, 1998.

Tsuboi, Y., K. Imanishi, K. Koyama, N. Grosso, and T. Montmerle, Quasiperiodic X-ray flares from the protostar YLW 15, ApJ, 532, 1089, 2000.

Yamauchi, S., K. Hamaguchi, K. Koyama, and H. Murakami, ASCA observations of the Chamaeleon II dark cloud, PASJ, 50, 465, 1998.

Zinnecker, H. and Th. Preibisch, X-ray emission from Herbig Ae/Be stars: A ROSAT survey, $A \& A, \mathbf{2 9 2}, 152,1994$.

K. Hamaguchi (e-mail: kenji@cr.scphys.kyoto-u.ac.jp), Y. Tsuboi, K. Imanishi, and K. Koyama 